\title{
Ru-Based Thin Film Temperature Sensor for Space Environments: Microfabrication and Characterization under Total Ionizing Dose
}

\author{
S. I. Ravelo Arias, ${ }^{1}$ D. Ramírez Muñoz, ${ }^{1}$ Susana Cardoso, ${ }^{2}$ and Paulo P. Freitas ${ }^{2,3}$ \\ ${ }^{1}$ Department of Electronic Engineering, University of Valencia, Avenida de la Universitat, s $n, 46100$ Burjassot, Spain \\ ${ }^{2}$ INESC Microsystems and Nanotechnologies (INESC-MN) and Physics Department, Instituto Superior Técnico, \\ Lisbon University, R. Alves Redol 9, 1000-029 Lisbon, Portugal \\ ${ }^{3}$ International Iberian Nanotechnology Laboratory (INL), Avenida Mestre José Veiga, 4715-31 Braga, Portugal
}

Correspondence should be addressed to D. Ramírez Muñoz; ramirez@uv.es

Received 15 December 2015; Revised 29 February 2016; Accepted 21 March 2016

Academic Editor: Oleg Lupan

Copyright (C) 2016 S. I. Ravelo Arias et al. This is an open access article distributed under the Creative Commons Attribution License, which permits unrestricted use, distribution, and reproduction in any medium, provided the original work is properly cited.

\begin{abstract}
The paper shows the microfabrication processes of a Ruthenium-based resistance temperature detector and its behavior in response to irradiation at ambient temperature. The radiation test was done in a public hospital facility and followed the procedures based on the ESA specification ESCC 22900. The instrumentation system used for the test is detailed in the work describing the sensors resistance evolution before, during, and after the exposure. A total irradiation dose of $43 \mathrm{krad}$ with $36 \mathrm{krad} / \mathrm{h}$ dose rate was applied and a subsequent characterization was performed once the Ru sensors were submitted to an $80^{\circ} \mathrm{C}$ annealing process during a period of $168 \mathrm{~h}$. The experimental measurements have shown the stability of this sensor against total ionizing dose (TID) tests, not only in their resistance absolute values during the irradiation phase but also in the relative deviation from their values before irradiation.
\end{abstract}

\section{Introduction}

Resistive thermometry is being used in science and industry since final decades of XIX century, as metals show predictable dependence of their electrical resistance on temperature. Platinum is the metal that combines high temperature linearity, repeatability, sensitivity, and stability. Resistive elements made of copper combine the highest linearity with temperature with poor robustness against corrosion as it starts to oxidize only above $100^{\circ} \mathrm{C}$. Nickel and its alloys bring about the advantage of their high temperature coefficient and low cost but worse linearity than platinum. In particular, this metal covers the widest range of temperature, from cryogenic values (liquid helium, $4.2 \mathrm{~K}$ ) to melting point (like gold, $1337 \mathrm{~K}$ ) [1].

When measuring temperature near absolute zero, the Ruthenium oxides have been used extensively because of their high sensitivity and resolution [2-6]. At ambient temperatures, metallic $\mathrm{Ru}$ has also been explored in a wide range of applications, as integrated on a compact water quality measurement system [7] or to compensate the thermal drift of magnetoresistive (MR) current sensors [8]. Particularly, these types of sensors are being used to measure and control the electrical current in spacecraft power systems [9]. At the same time, accurate thermal control of various sections of the equipment used on board minimizes failures and power consumption.

Batteries, propulsion subsystems, generic electronics, or infrared channels must be thermally controlled within their operation range, which includes a temperature interval comprised between $120 \mathrm{~K}$ and $420 \mathrm{~K}[10,11]$. Ruthenium temperature sensors can be directly integrated in the electronic circuits as a thin film form, therefore bringing about an alternative to be used in the thermal control units of spacecraft systems.

The present work describes an experimental methodology to investigate the stability of $\mathrm{Ru}$ thin film temperature sensors under irradiation, following the mandatory protocol for evaluating sensors and electronics components prior to 


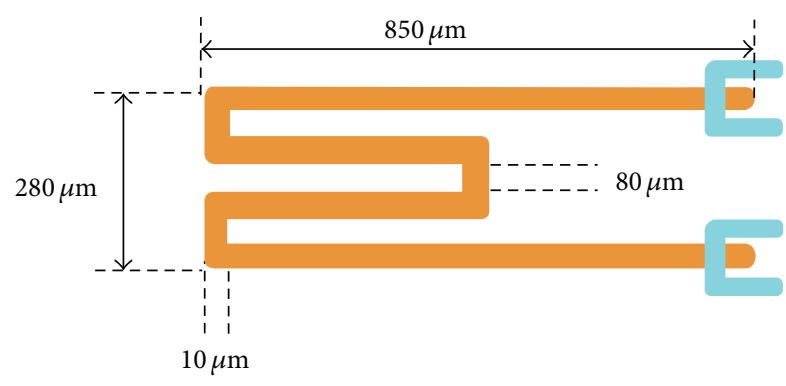

FIGURE 1: Relevant dimensions of the Ru sensor film.

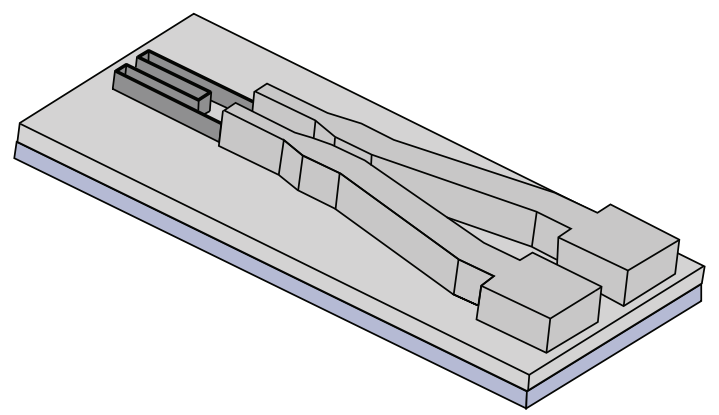

FIGURE 2: Connections pattern definition of the Ru sensor after liftoff and cleaning steps.

their onboard integration in space missions. The European Space Agency (ESA) procedure described in the specification ESCC 22900 was used in its main statements [12]. As a result, the feasibility to use $\mathrm{Ru}$ films as temperature sensors in spacecraft environments is assessed through their stability against a total ionizing dose (TID).

\section{Device Fabrication and Layout}

Silicon $\langle 100\rangle 650 \mu \mathrm{m}$ thick wafer was used as substrate for the sensors. A $1800 \AA$ thick $\mathrm{Al}_{2} \mathrm{O}_{3}$ layer was deposited by RF magnetron sputtering ( $200 \mathrm{~W}, 45 \mathrm{sccm}$ Ar, $3.8 \mathrm{mTorr}$ ) for electrical insulation. Then, the temperature sensors were defined by laser lithography (DWL Lasarray 2.0, $442 \mathrm{~nm}$ laser beam) with the geometry shown in Figure 1, where the $850 \mu \mathrm{m} \times 280 \mu \mathrm{m}$ area was used to imprint the serpentine layout defining the active sensor.

A $400 \AA$ thick Ru film was then deposited by ion beam deposition process (IBD, Nordiko 3600 tool [13]) and defined by lift-off. Electrical contacts consist of a $3000 \AA$ thick $\mathrm{AlSiCu}$ metal layer deposited by magnetron sputtering (Nordiko 7000 machine, $2 \mathrm{~kW}, 3$ mTorr, $50 \mathrm{sccm}$ Ar) and protected with $150 \AA$ thick $\mathrm{TiWN}_{2}$ films. The subsequent lift-off and cleaning steps defined the connections pattern shown schematically in Figure 2.

Finally, passivation was accomplished using a $3000 \AA$ thick silicon nitride $\left(\mathrm{Si}_{3} \mathrm{~N}_{4}\right)$ dielectric layer deposited by plasma-assisted chemical vapor deposition (electrotech machine, at $300^{\circ} \mathrm{C}, 850$ mTorr with $\mathrm{SiH}_{4}, \mathrm{NH}_{3}$, and $\mathrm{N}_{2}$ gases flux) over the wafer, using reactive plasma-etching (LAM machine ion-enhanced fluorine-based plasma, at 140 mTorr

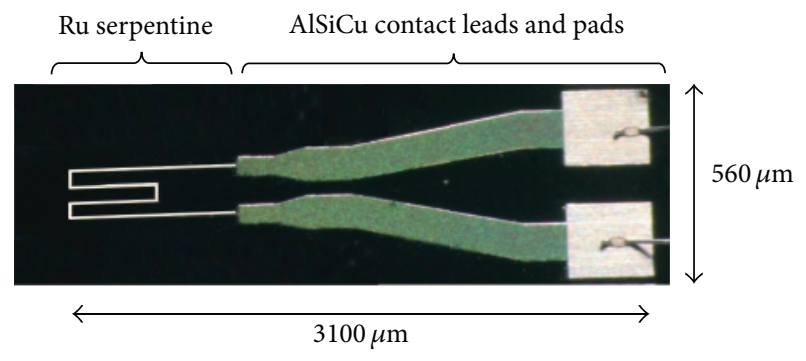

FIGURE 3: Actual layout of the Ru temperature sensor.

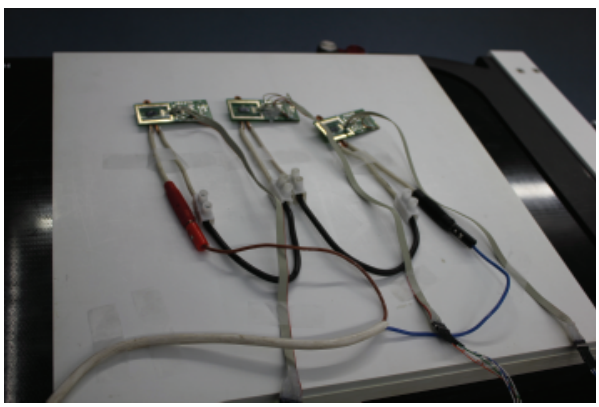

FIGURE 4: Ru sensors placed in the irradiation area.

with $\mathrm{Ar}, \mathrm{CF}_{4}$, and $\mathrm{O}_{2}$ gases flux) technique to define the contact pads holes over the dielectric layer. Figure 3 shows the actual layout of the microfabricated Ru sensor.

\section{Experimental Methodology}

The irradiation procedure followed the ESA specification [12] and was carried out at the facility of the Radiotherapy Department within the La Fe University Hospital in Valencia (Spain).

In the experiment, three $\mathrm{Ru}$ thin film sensors were irradiated by an X-rays photons beam produced by the source of accelerated electrons. Figure 4 shows the target area $(40 \mathrm{~cm} \times 40 \mathrm{~cm})$ enclosing the printed-circuit-board where the $\mathrm{Ru}$ sensors were placed.

The beam consisted of a photons spectrum up to $6 \mathrm{MeV}$ of energy with an average of $2 \mathrm{MeV}$ and a radiation dose of $36 \mathrm{krad} / \mathrm{h}(6 \mathrm{~Gy} / \mathrm{min})$. That value was a compromise between the standard dose used at the hospital facility and the rate group considered in the ESA specification [12]. Also, a maximum irradiation level of $43 \mathrm{krad}$ was decided as the optimum dose taking into account the time needed by the irradiation facility for the patients therapy. That dose level sets the room usage to less than $80 \mathrm{~min}$, being enough to accomplish the $43 \mathrm{krad}$ level identified as the D class in [12]. The ambient temperature during the experiment was stable at $19.5^{\circ} \mathrm{C}$ fulfilling either the requirement of $t=20 \pm 10^{\circ} \mathrm{C}$ or the specification that variations should be lower than $3^{\circ} \mathrm{C}$ during the irradiation time.

The specification described in [12] stated that sensors under test must be characterized once the irradiation exposure finishes. In particular, the $\mathrm{Ru}$ sensor resistance was measured at the same temperature after 12,24 , and $168 \mathrm{~h}$ of 


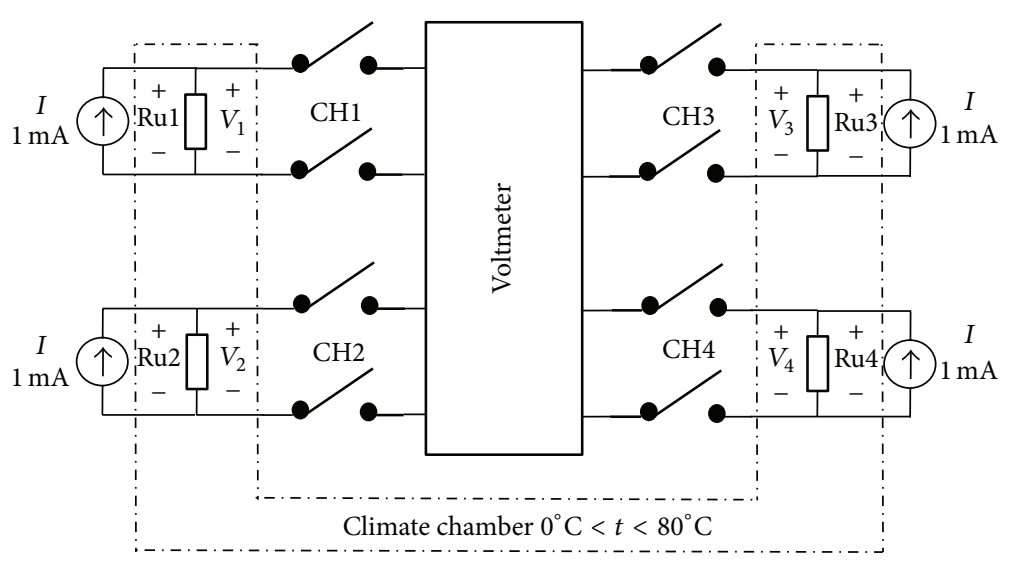

FIgURE 5: Measurement and acquisition system designed to characterize the $\mathrm{Ru}$ sensors (voltmeter and switches $\mathrm{CH} 1$ to $\mathrm{CH} 4$ were implemented by the unit 34970A from Agilent).

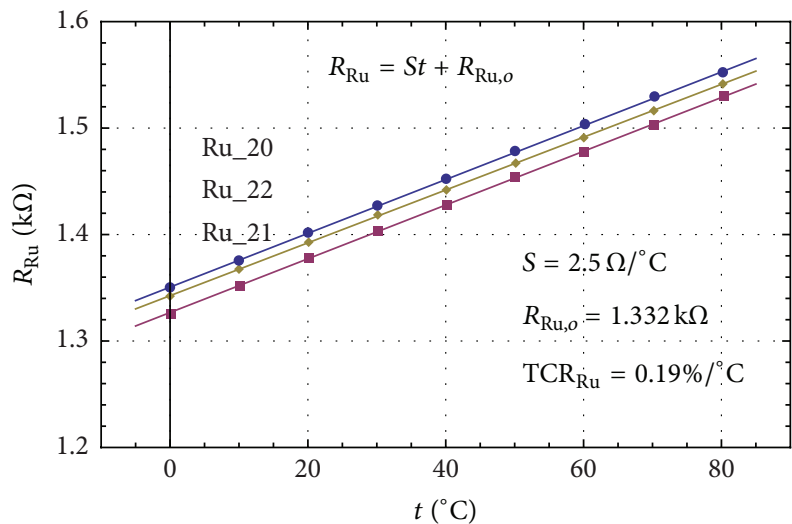

FIGURE 6: Experimental resistance-to-temperature characteristic of the $\mathrm{Ru}$ samples $\mathrm{Ru} \_20, \mathrm{Ru} 221$, and $\mathrm{Ru} \_22$ before irradiation.

total irradiation time. Afterwards, the sensors were submitted to an annealing process of $168 \mathrm{~h}$ at $80^{\circ} \mathrm{C}$ and finally their resistance was measured once again at the initial temperature of $19.5^{\circ} \mathrm{C}$. To pass or fail the test, stability criteria of $1^{\circ} \mathrm{C}$ as a maximum variation during the exposure time were assumed.

Before submitting the $\mathrm{Ru}$ sensors to the irradiation exposure, a temperature characterization was done to know the Ru temperature coefficient, $\mathrm{TCR}_{\mathrm{Ru}}$, its temperature sensitivity, $S$, and the reference resistance at $0^{\circ} \mathrm{C}, R_{\mathrm{Ru}, o}$. To do this, the sensors resistance was obtained measuring the voltage drop across their terminals when circulating a $1 \mathrm{~mA}$ DC current. Previous works with similar sensors found a thermal resistance of about $0.06^{\circ} \mathrm{C} / \mathrm{mW}$ driving with $1 \mathrm{~mA}$. Considering that value, the temperature deviation due to self-heating was estimated less than $0.02 \%$. In a specific application, the driving current would be lesser. Simultaneously, the sensors were submitted inside a climate chamber to a temperature sweep from $0^{\circ} \mathrm{C}$ to $80^{\circ} \mathrm{C}$ in steps of $10^{\circ} \mathrm{C}$. Figure 5 shows the acquisition system designed to measure the voltage across each of the $\mathrm{Ru}$ sensors once they were supplied with a $1 \mathrm{~mA}$ independent current source. In this experiment, the voltmeter and switches $\mathrm{CH} 1$ to $\mathrm{CH} 4$ were implemented by the unit 34970A from Agilent. The resistance

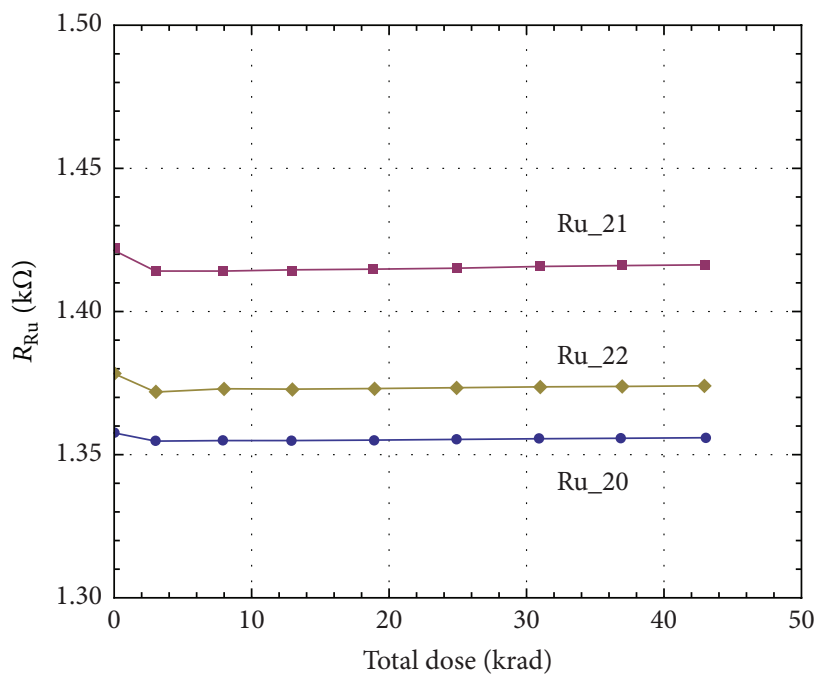

FIGURE 7: Resistance dependence under increasing irradiation doses, for the $3 \mathrm{Ru}$ sensors.

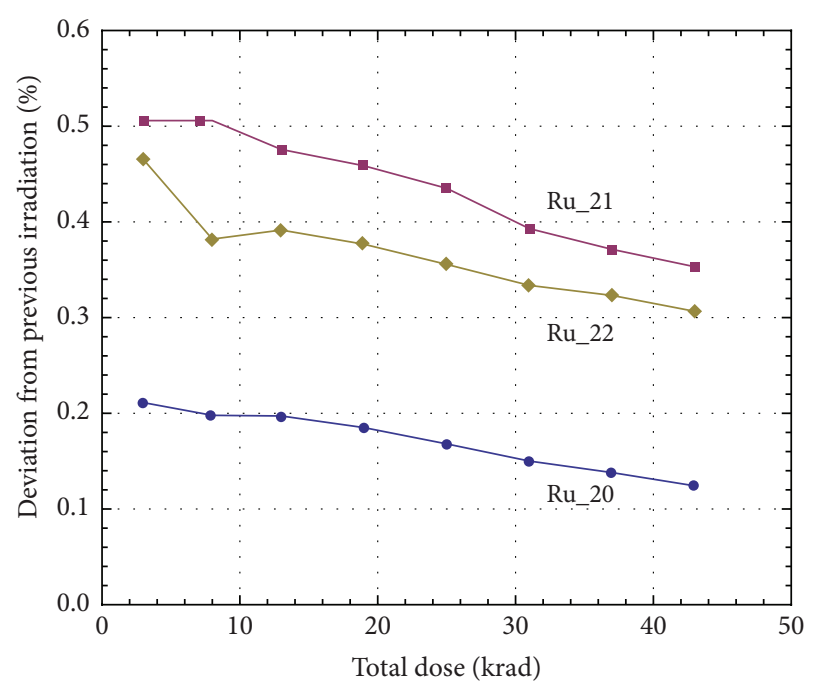

FIGURE 8: Percentage change of the irradiated Ru sensors compared with the initial nonirradiated one. 


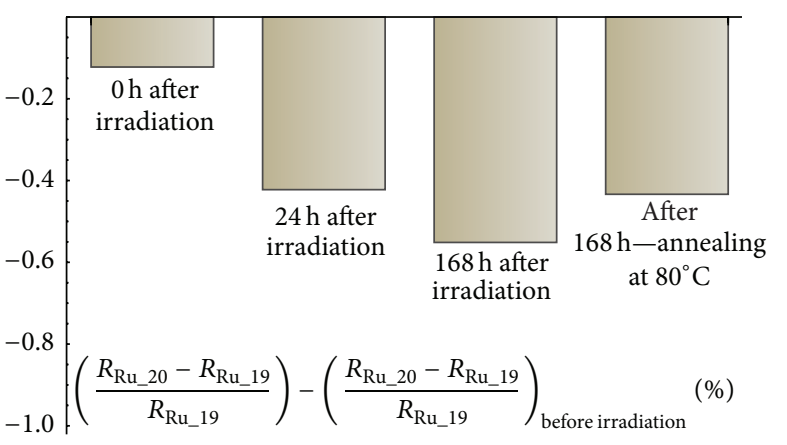

(a)

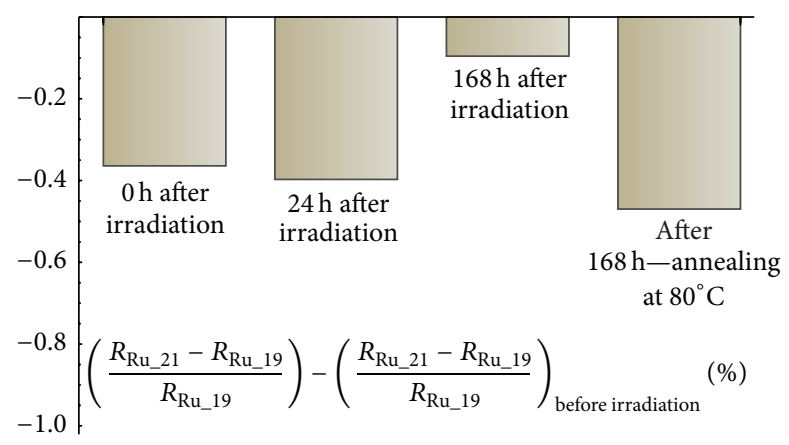

(b)

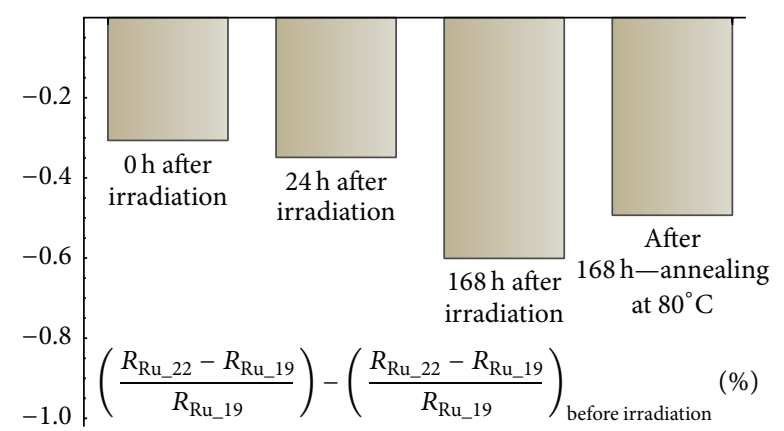

(c)

FIGURE 9: Variation of the relative deviation of Ru resistance with respect to the reference sensor: (a) Ru_20, (b) Ru_21, and (c) Ru_22 samples.

measurements uncertainty was obtained from an electronic current source implemented with a reference voltage $V_{\text {ref }}$ and a precision resistor $R$, both components with a relative uncertainty of about $0.1 \%$. The voltage measurements across the sensors were taken using the unit 34970A from Agilent. This equipment has a $6^{1 / 2}$-digit built-in digital voltmeter. Doing several calculations, these data made an uncertainty of $0.15 \%$ for the sensors resistance measurements.

Figure 6 shows the experimental resistance-to-temperature characteristic of the three $\mathrm{Ru}$ sensors with their average temperature coefficients, from which a very good linearity was obtained in that temperature range.

\section{Experimental Results and Discussion}

Once the resistance-to-temperature characteristic was obtained in the absence of irradiation, the resistance of the sensors was measured during the irradiation time as the total dose was increased (Figure 7). Figure 8 shows the deviation with respect to the initial nonirradiated value, from which a high stability can be obtained, with maximum variation of $0.5 \%, 0.46 \%$, and $0.2 \%$, respectively, for samples $\mathrm{Ru}_{2} 20$, $\mathrm{Ru} \_21$, and $\mathrm{Ru} \_22$. The temperature variation associated with the irradiation time was of $0.5^{\circ} \mathrm{C}, 0.9^{\circ} \mathrm{C}$, and $0.9^{\circ} \mathrm{C}$, respectively; these values were less than the stability criteria assumed initially.

Following the procedure described in the previous section, the Ru sensors resistance was obtained at 12, 14, and $168 \mathrm{~h}$ after the irradiation time and at the end of an additional $168 \mathrm{~h}$ annealing period at $80^{\circ} \mathrm{C}$. All the resistance measurements were taken at the same initial temperature of the experiment $\left(19.5^{\circ} \mathrm{C}\right)$.

Figure 9 shows how the relative deviation in \% of each irradiated sensor with respect to the reference one varies from the initial \% before irradiation. It can be concluded that the measurements done reveal that the Ru electrical resistance, apart from this optimum linearity as a temperature sensor, has a strong stability against total ionizing dose tests. The sensors manifest slight variation not only in their resistance absolute values during the irradiation phase but also in their relative deviation from the previous irradiation values. The electrical resistance stability can be extended when comparing, as basic specification [12] states, the relative deviation with respect to a reference sensor before and after irradiation.

\section{Conclusions}

The aim of the work was to demonstrate the feasibility of thin film $\mathrm{Ru}$ resistors as temperature detectors under irradiation exposure. The validation was carried out under the protocol described in the ESA ESCC basic specification 22900. The measurements obtained by the designed instrumentation system revealed excellent stability with the irradiation doses (less than $0.5 \%$ variation up to $40 \mathrm{krad}$ ). The observed sensor behavior remained stable (less than $0.7 \%$ variation) after a $168 \mathrm{~h}$ annealing period at $80^{\circ} \mathrm{C}$. As a consequence, Ru-based resistance temperature sensors offer an interesting choice to use in space instrumentation and control systems requiring integrated temperature measurement at the conventional 
range (from $120 \mathrm{~K}$ to $420 \mathrm{~K}$ ). Further research is being done combining total ionizing doses and cryogenic temperatures to evaluate the $\mathrm{Ru}$ sensors feasibility to be applied in cryogenic instruments or systems, integrated with the electronic components.

\section{Competing Interests}

The authors declare that they have no competing interests.

\section{Acknowledgments}

The authors would like to thank Dr. J. Pérez Calatayud, Director of the Radiotherapy Service at the La Fe Hospital (Valencia, Spain), for his kindness to provide the hospital facilities for this work. This work was supported in part by the Spanish Ministry of Economics and Competitivity under the AYA2012-37444-C02-01 Project by the Generalitat Valenciana under the Prometeo/2012/044 Project and by the 217152-312630 Grant of the Consejo Nacional de Ciencia y Tecnología (CONACYT México). INESC-MN acknowledges FCT funding through Project EXCL/CTM-NAN/0441/2012 and IN Associated Laboratory (Pest-OE/CTM/LA0024/ 2011).

\section{References}

[1] W. Göpel, J. Hesse, and J. N. Zemel, Eds., Sensors, VCH Press, Weinheim, Germany, 1990.

[2] R. W. Willekers, F. Mathu, H. C. Meijer, and H. Postma, “Thick film thermometers with predictable R-T characteristics and very low magnetoresistance below $1 \mathrm{~K}$," Cryogenics, vol. 30, no. 4, pp. 351-355, 1990.

[3] I. Bat'ko, K. Flachbart, M. Somora, and D. Vanický, "Design of $\mathrm{RuO}_{2}$-based thermometers for the millikelvin temperature range," Cryogenics, vol. 35, no. 2, pp. 105-108, 1995.

[4] J. Ylöstalo, P. Berglund, T. O. Niinikoski, and R. Voutilainen, "Cryogenic temperature measurement for large applications," Cryogenics, vol. 36, no. 12, pp. 1033-1038, 1996.

[5] G. G. Ihas, L. Frederick, and J. P. McFarland, "Low temperature thermometry in high magnetic fields," Journal of Low Temperature Physics, vol. 113, no. 5-6, pp. 963-968, 1998.

[6] R. Sahul, V. Tasovski, and T. S. Sudarshan, "Ruthenium oxide cryogenic temperature sensors," Sensors and Actuators A: Physical, vol. 125, no. 2, pp. 358-362, 2006.

[7] S. Zhuiykov, D. O’Brien, and M. Best, "Water quality assessment by an integrated multi-sensor based on semiconductor $\mathrm{RuO}_{2}$ nanostructures," Measurement Science and Technology, vol. 20, no. 9, Article ID 095201, 2009.

[8] J. S. Moreno, D. R. Muñoz, S. Cardoso, S. C. Berga, A. E. N. Antón, and P. J. P. de Freitas, "A Non-invasive thermal drift compensation technique applied to a spin-valve magnetoresistive current sensor," Sensors, vol. 11, no. 3, pp. 2447-2458, 2011.

[9] R. Slatter and B. Goffin, "Magnetoresistive current sensors for high accuracy, high bandwidth current measurement in spacecraft power electronics," in Proceedings of the European Space Power Conference, Noordwijkerhout, The Netherlands, April 2014.

[10] M. N. de Parolis and W. Pinter-Krainer, "Current and future techniques for spacecraft thermal control 1. Design drivers and current technologies,' ESA Bulletin 87, European Space Agency, Paris, France, 1996.

[11] NASA, Goes I-M Databook, DRL 101-08, Rev. 1, 1996.

[12] ESCC, "Total dose steady-state irradiation test method," ESAESCC Basic specification no. 22900, issue 4, ESCC, 2010.

[13] R. Ferreira, S. Cardoso, P. P. Freitas, R. Petrova, and S. McVitie, "Influence of ion beam assisted deposition parameters on the growth of $\mathrm{MgO}$ and CoFeB," Journal of Applied Physics, vol. 111, no. 7, Article ID 07C117, 2012. 


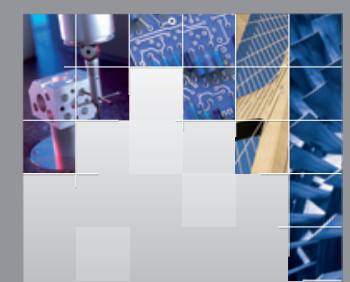

\section{Enfincering}
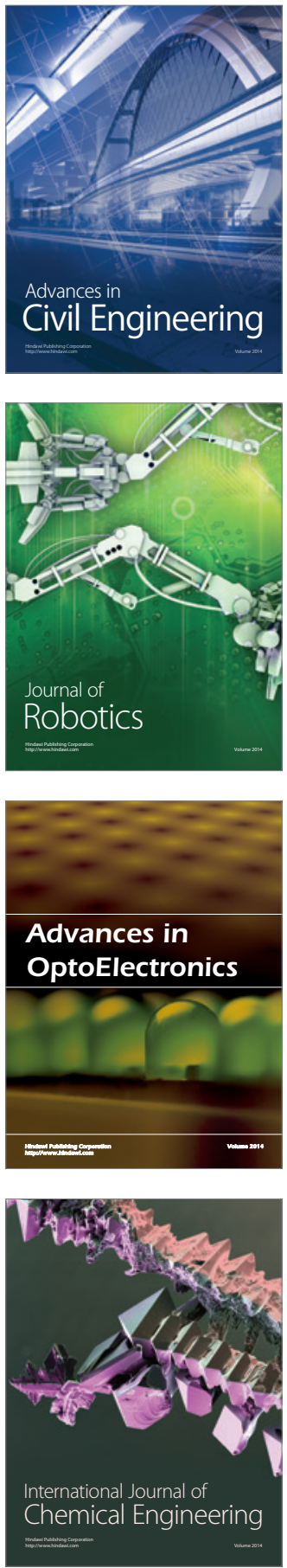

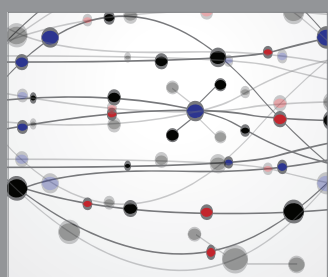

The Scientific World Journal

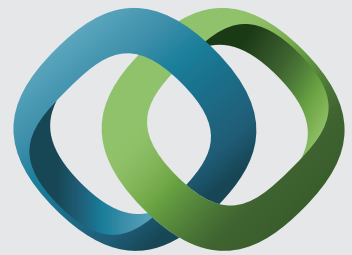

\section{Hindawi}

Submit your manuscripts at

http://www.hindawi.com
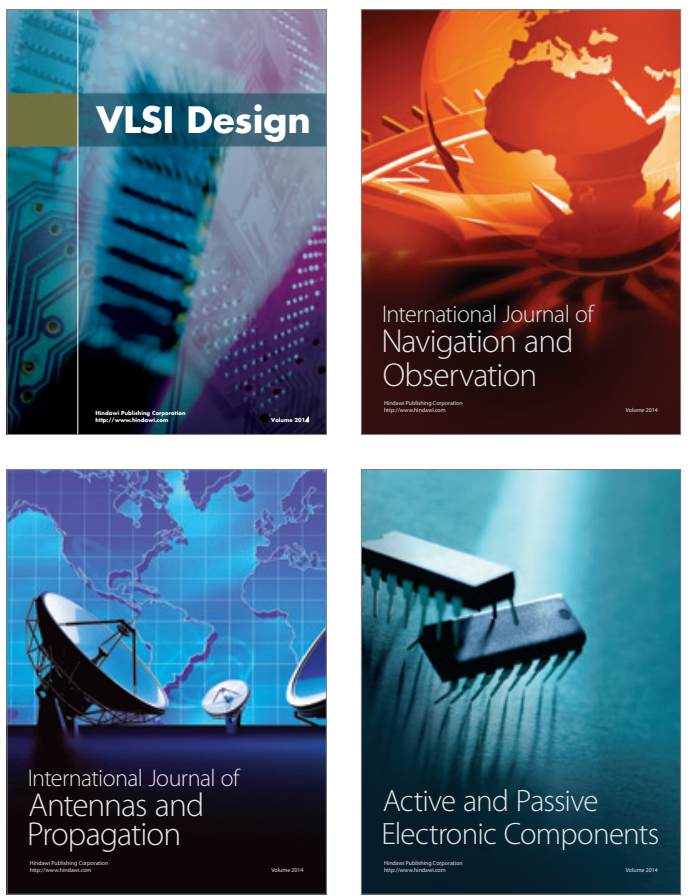
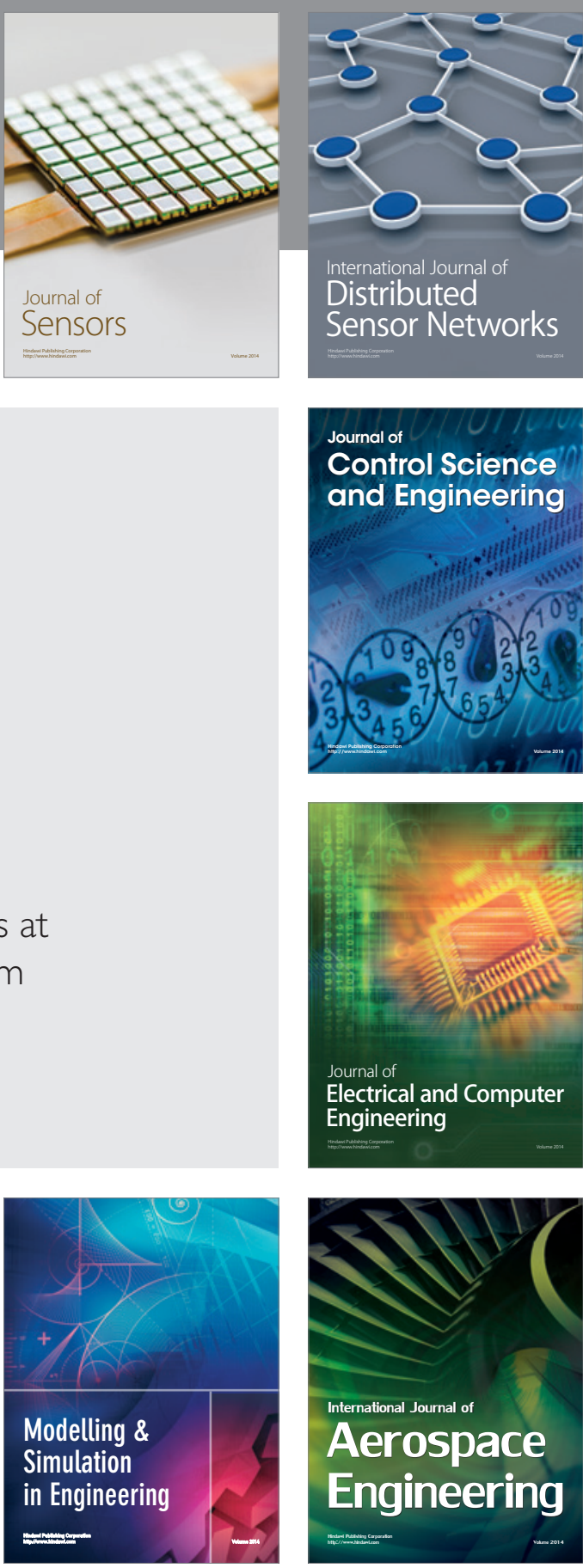

International Journal of

Distributed

Sensor Networks

Journal of

Control Science

and Engineering
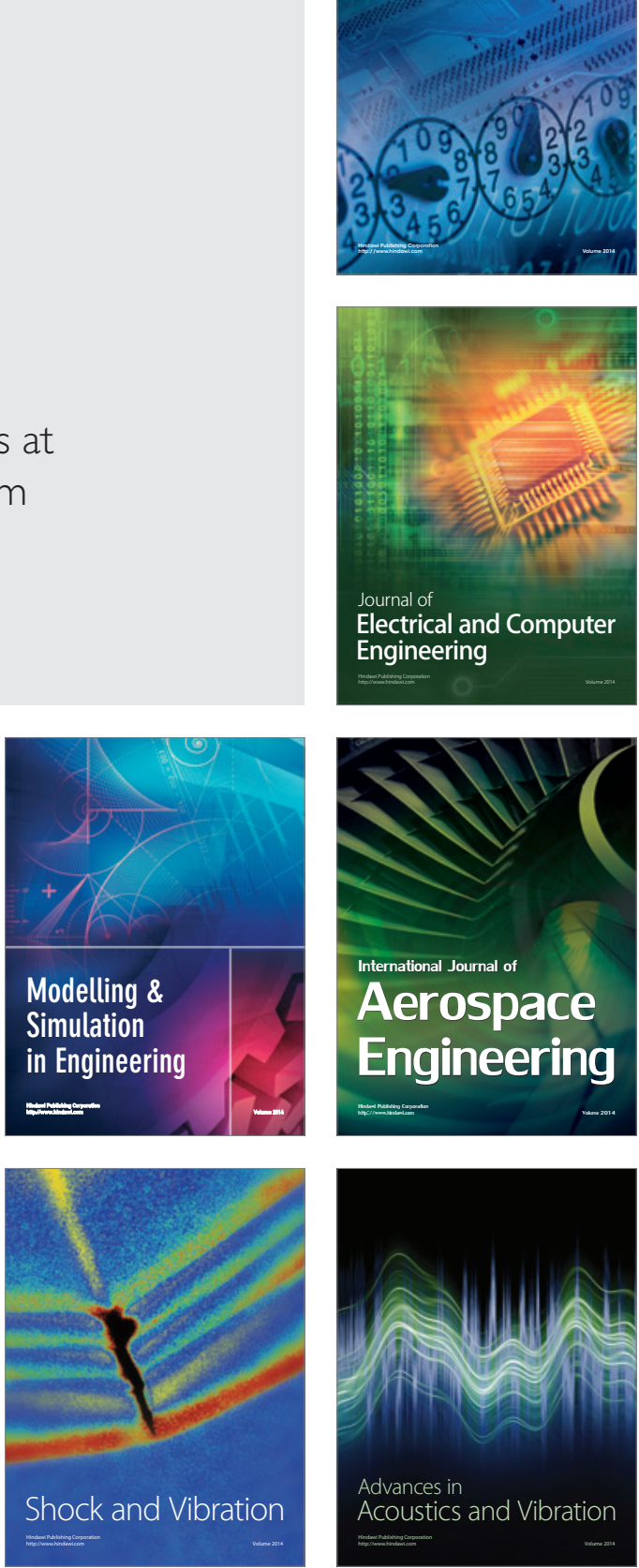